Сенво П.

\title{
СИСТЕМНИЙ АНАЛІЗ
}

УДК 517.26:517.928.1:519.615.3

\section{СПЕЦИФІКА МАТЕМАТИКИ БАГАТОВИМІРНИХ ФУНКЦІОНАЛЬНИХ ІНТЕРВАЛІВ}

\section{П. Сеньо}

Львівсъкий національний університет імені Івана Франка, вул. Університесъка, 1, Львів, 79000, e-mail: petrosny@ukr.net

\begin{abstract}
Розглянуто розроблену автором математику функціональних інтервалів в $R^{n}, n>$ 1, виокремлено специфічні моменти в ній у побудові функціональних інтервалів функцій багатьох змінних, арифметичних, унарних, теоретико-множинних операцій та запропоновано ефективні алгоритми їхньої реалізації. Для цього використано метод декомпозиції та спрощену математику функціональних інтервалів у $R^{1}$. Це зумовлено тим, що результати виконання арифметичних операцій над функціональними інтервалами визначаються відповідними операціями над їхніми обмежувальними функціями [2]. Але результати виконання арифметичних операцій над лінійними функціями є відповідними лінійними функціями лише у разі додавання та віднімання таких функцій. Крім того, навіть в $R^{2}$ результати множення та ділення таких функцій можуть містити, наприклад, сідлові точки, що не дає змоги апроксимувати їх лінійними функціями. Отже, в таких випадках множина результатів дій над багатовимірними функціональними інтервалами не $є$ замкненою. Описана проблема розв'язана застосуванням спрощеної математики функціональних інтервалів по всіх координатах, крім однієї, яку послідовно змінюємо від 1 до $n$. Тоді всі арифметичні операції над багатовимірними функціональними інтервалами зводяться до відповідних операцій над двома одновимірними функціональними інтервалами, обмежувальні функції одного з яких є кусково-постійними функціями. Лінійний функціональний інтервал цієї функції багатьох змінних на зазначеному багатовимірному інтервалі $є$ перетином всіх так побудованих одновимірних функціональних інтервалів і будь-яких вибірок із них. Для знаходження таких перетинів потрібно розв'язувати відповідні системи лінійних алгебричних рівнянь.
\end{abstract}

Ключові слова: інтервал, функціональний інтервал, інтервальне розширення функції, двостороння апроксимація, математика функціональних інтервалів.

\section{1. ВСТУП}

Задача побудови двосторонніх апроксимацій функцій дуже актуальна. Сучасні методи розв'язання цієї проблеми, головно засновані на інтервальній математиці. Вони мають широкий спектр застосувань і в багатьох випадках дають вичерпні рекомендації щодо вибору стратегії та методів наближених обчислень. Близькі до них двосторонні обчислювальні методи, які також належать до прямих методів оцінки точності похибки обчислень.

Зазвичай в інтервальному аналізі задачу побудови двосторонніх апроксимацій функцій розв'язують за допомогою побудови інтервальних розширень заданих функцій [1].Ефективність методів розв'язування задач, розроблених на основі інтервального аналізу, суттєво залежить від близькості відповідних природних інтервальних розширень функцій, які входять у математичну модель задачі, до їхніх об'єднаних інтервальних розширень. Тому багато праць спрямовано на розробку методів побудови оптимальних природних розширень функцій [2]. Але навіть

(C) Сеньо П., 2020 
цілковитий збіг обох цих інтервальних розширень мінімізує величину відхилення значення функції від меж інтервалу - інтервального розширення функції лише у точках її глобальних екстремумів.

У [3] проблема мінімізації величин відхилення значення функції від меж i двосторонніх апроксимацій розв'язана у $R^{1}$ за допомогою математики функціональних інтервалів.

\section{2. ФОРМУЛЮВАННЯ ЗАДАЧІ}

У [3] для функцій одного аргументу запропоновано алгоритм побудови функціональних інтервалів на основі нерівності Єнсена. Він суттєво використовує монотонність та опуклість цієї функції. В такому випадку для побудови їі лінійного функиіонального інтервалу [3] використовуємо дотичну в кінці або в середині інтервалу, та січну.

Якщо задана функція на інтервалі $X$ немонотонна і неопукла, то попередньо цей інтервал треба розбити на інтервали, де ця функція володіє такими властивостями. Для цього треба знати у інтервалі $X$ точки його кінців, всі точки екстремумів, точки перегинів, розривів; точки кінців інтервалів, де функція задана різними аналітичними виразами; точки недиференційовності цієї функції. Ці точки будемо називати характерними точками заданої функції. Така інформація відома, зокрема, для елементарних, типових і спеціальних функцій. Тоді на кожному так отриманому інтервалі побудову лінійного функціонального інтервалу виконуємо за допомогою січних і дотичних за наведеним нижче алгоритмом. Він враховує те, що графік функції з постійною опуклістю розміщений по один бік від будь-якої дотичної, проведеної до нього, і по інший бік від його січної. Тому маємо такий алгоритм.

Алгоритм 1. Нехай $x_{1} \leq x_{2} \leq \ldots \leq x_{n}$, де $x_{i} \in X, i=\overline{1, n}$, характерні точки заданої функції $y=g(x)$ у інтервалі $X$. Тоді на кожному інтервалі $X_{i}=\left[x_{i}, x_{i+1_{i}}\right]$ функція монотонна і має постійну опуклість (або опукла вверх, або опукла вниз).

1. Якщо на інтервалі $X_{i}$ задана функція опукла вверх, то верхня обмежувальна функція $\bar{l}_{g}^{(i)}(x)$ лінійного функціонального інтервалу $L_{g}^{(i)}=\left\{X_{i}, \underline{l}_{g}^{(i)}(x), \bar{l}_{g}^{(i)}(x)\right\}$ складається з кусків дотичних, проведених у точках $x_{i}, x_{i+1}$ до графіка функції $y=g(x)$ до їх перетину; нижня його обмежувальна функція $\underline{l}_{g}^{(i)}(x)$ складається з кусків січних, проведених у точках $x_{i}, x_{i+1}$ до графіка цієї функції та точки перетину проведених дотичних.

2. Якщо на інтервалі $X_{i}$ задана функція опукла вниз, то нижня обмежувальна функція $\underline{l}_{g}^{(i)}(x)$ лінійного функціонального інтервалу $L_{g}^{(i)}=\left\{X_{i}, \underline{l}_{g}^{(i)}(x), \bar{l}_{g}^{(i)}(x)\right\}$ складається із кусків дотичних, проведених у точках $x_{i}, x_{i+1}$ до графіка функції $y=g(x)$ до їх перетину; верхня його обмежувальна функція $\bar{l}_{g}^{(i)}(x)$ складається із кусків січних, проведених у точках $x_{i}, x_{i+1}$ до графіка цієї функції та точки перетину проведених дотичних.

3. Після побудови всіх лінійних функціональних інтервалів $L_{g}^{(i)}=\left\{X_{i}, \underline{l}_{g}^{(i)}(x)\right.$, $\left.\bar{l}_{g}^{(i)}(x)\right\}, i=\overline{1, n-1}$, лінійний функціональний інтервал функції $y=g(x)$ на всьому інтервалі $X$ отримуємо, об'єднавши так побудовані лінійні функціональні інтервали $L_{g}^{(i)}$, тобто, $L_{g}=\bigcup_{i=1}^{n-1} L_{g}^{(i)}$.

Зауваження 1. Для побудови лінійного функціонального інтервалу кожної функції всі ї̈ характерні точки обов'язково мають входити у множину точок розбиття інтервалу $X$. Однак для звуження двосторонньої апроксимації цієї функції 
Сенво П

ISSN 2078-5097. Вісн. Львів. ун-ту. Сер. прикл. матем. та інф. 2020. Вип. 28

обмежувальними функціями лінійного функціонального інтервалу, множину точок розбиття інтервалу $X$ можна доповнити бажаною кількістю інших точок з цього інтервалу.

У багатовимірних просторах безпосередньо застосувати зазначений алгоритм неможливо. Тому це суттєво ускладнює реалізацію всіх арифметичних, унарних i теоретикомножинних операцій та породжує специфіку математики багатовимірних інтервалів.

\section{3. ЗАГАЛЬНА СХЕМА РОЗВ'ЯЗАННЯ ЗАДАЧІ}

\section{1. СПРОЩЕНА МАТЕМАТИКА ЛІНІЙНИХ ФУНКЦІО- НАЛЬНИХ ІНТЕРВАЛІВ У $R^{1}$}

Загальна схема розв'язання задачі. Специфіка багатовимірних функціональних інтервалів і операцій над ними потребує для розв'язування проблеми мінімізації величини відхилення значення функції багатьох змінних від меж їі двосторонніх апроксимацій принципово іншого підходу. У цьому випадку використовуємо метод декомпозиції та спрощену математику функціональних інтервалів у $R^{1}$.

Неоднозначність визначення функціональних інтервалів дає змогу побудувати спрощену математику лінійних функціональних інтервалів. У ній лінійні функціональні інтервали будуються не за допомогою січних і дотичних цієї функції, а за допомогою прямих, паралельних осі абсцис. Це можливо зробити лише для функцій, всі характерні точки яких відомі. Такими, зокрема, є елементарні, типові та спеціальні функції.

Нехай у інтервалі $X$ відомі всі характерні точки $x_{i} \in X, i=\overline{1, n}$ заданої функції $y=g(x)$, причому $x_{1} \leq x_{2} \leq \ldots \leq x_{n}$. Отже, на кожному інтервалі $X_{i}=\left[x_{i}, x_{i+1_{i}}\right]$ ця функція монотонна і має постійну опуклість. Тому на цих інтервалах графік функції розташований по один бік від горизонтальної прямої, що проходить через точку $\left(x_{i}, y_{i}\right)$, де $y_{i}=g\left(x_{i}\right)$. Оскільки у цій математиці кутові коефіцієнти всіх обмежувальних функцій елементарних лінійних функціональних інтервалів дорівнюють нулю, то зміщення $\underline{m}, \bar{m}$ у їхніх обмежувальних функціях дорівнюють значенням функції $y=g(x)$ у її характерних точках. Якщо на проміжку $\left[x_{i}, x_{i+1_{i}}\right]$ ця функція зростає, то їі лінійний функціональний інтервал $G\left(\left[x_{i}, x_{i+1}\right]\right)=$ $\left\{\left[x_{i}, x_{i+1}\right], y_{i}, y_{i+1}\right\}$, а якщо спадає, то $G\left(\left[x_{i}, x_{i+1}\right]\right)=\left\{\left[x_{i}, x_{i+1}\right], y_{i+1}, y_{i}\right\}$.

Оскільки на кожному інтервалі $\left[x_{i}, x_{i+1_{i}}\right]$ всі перетини лінійного функціонального інтервалу функції $y=g(x)$ є тим самим інтервалом $\left(\left[y_{i}, y_{i+1}\right]\right.$, чи $\left.\left[y_{i+1}, y_{i}\right]\right)$, то всі арифметичні і теоретико-множинні операції над такими лінійними функціональними інтервалами є відповідними операціями інтервальної математики, які зводяться до цих же операцій зі значеннями цієї функції у її характерних точках. Тому і відстань між такими елементами, норма і ширина елементарного лінійного функціонального інтервалу є інтервальною відстанню, нормою та шириною, відповідно.

Зауваження 3.1. Інтервальне розширення функції значно ширше навіть від їі лінійного функціонального інтервалу, обчисленого з використанням спрощеної математики лінійних функціональних інтервалів. 


\section{2. МАТЕМАТИКА БАГАТОВИМІРНИХ ФУНКЦІО- НАЛЬНИХ ІНТЕРВАЛІВ}

Означення 3.1. Багатовимірним функціональним інтервалом, багатовимірним функціональним обмежником, визначеним у інтервалі $X$, де $X=X_{1} \times X_{2} \times \ldots X_{n}$, будемо називати параметризовану множину інтервалів $\left[f_{1}(x), f_{2}(x)\right]$, де $f_{1}, f_{2}: X \subset$ $R^{n} \rightarrow R^{1}, f_{1}(x) \leq f_{2}(x)$, параметром якої $\epsilon$ аргумент $x \in X, i$ позначимо його, відповідно, $F(X)$, або $\left\{X, f_{1}(x), f_{2}(x)\right\}$.

Отже, багатовимірний функціональний інтервал - це множина всіх точок частини $n+1$ - вимірного простору, що на інтервалі $X$ розташовані між множинами значень функцій $f_{1}\left(x_{1}, x_{2}, \ldots, x_{n}\right), f_{2}\left(x_{1}, x_{2}, \ldots, x_{n}\right)$, включно з ними, де $f_{1}(x) \leq f_{2}(x)$.

Означення 3.2. Частинним перетином першого порядку функціонального інтервалу $\left\{X, f_{1}(x), f_{2}(x)\right\}$ називатимемо $n$ - вимірний функціональний інтервал $\left\{X_{1} \times X_{2} \times \ldots \times X_{i-1} \times X_{i+1} \times \ldots \times X_{n}, f_{1}(x), f_{2}(x)\right\}$, у якому значення обмежувалвних функиій обчислені при фіксованій одній координаті $x_{i}$ точки $x$.

Аналогічно визначаються і частинні перетини вищих порядків функціонального інтервалу.

Означення 3.3. Реалізацією функціонального інтервалу $\left\{X, f_{1}(x), f_{2}(x)\right\}$ називатимемо кожну функцію $f(x)$, значення якої у інтервалі $X$ належить цьому функціональному інтервалу, тобто $f_{1}(x) \leq f(x) \leq f_{2}(x)$. Функціі $f_{1}(x), f_{2}(x)$ називатимемо обмежувальними функціями цього функціонального інтервалу. Якщо однією з реалізацій функціонального інтервалу $\left\{X, f_{1}(x), f_{2}(x)\right\}$ є функція $f(x)$, то цей функціональний інтервал будемо називати функціональним інтервалом функції $f(x)$.

Отже, функціональний інтервал можна також інтерпретувати як множину всіх відповідних функцій - реалізацій. Це свідчить про зв'язок функціональних інтервалів з стохастичними процесами.

Якщо функції $f_{1}(x)$ і $f_{2}(x)$ - міноранта та мажоранта функції $f(x)$, відповідно, то функціональний інтервал $\left\{X, f_{1}(x), f_{2}(x)\right\}$ містить всі значення функції $f(x)$, якщо $x \in X$. Тому інтервальне розширення $\left[c_{1}, c_{2}\right]$ функції $f(x) \in$ частинним випадком функціонального інтервалу $\left\{X, f_{1}(x), f_{2}(x)\right\}$.

\section{3. АРИФМЕТИЧНІ ТА ТЕОРЕТИКО-МНОЖИННІ ОПЕ- РАЦІЇ $З$ БАГАТОВИМІРНИМИ ФУНКЦІОНАЛЬНИ- МИ ІНТЕРВАЛАМИ}

Арифметичні та теоретико-множинні операції з багатовимірними функціональними інтервалами виконуються аналогічно до відповідних означень з [3]. Зокрема, аналогічно до них отримуємо такі формули операцій додавання і віднімання елементарних багатовимірних лінійних функціональних інтервалів $\left\{X, \underline{l}^{(1)}(x), \bar{l}^{(1)}(x),\right\}$ i $\left\{X, \underline{l}^{(2)}(x), \bar{l}^{(2)}(x),\right\}:$

$$
\begin{gathered}
\left\{X, \underline{l}^{(1)}(x), \bar{l}^{(1)}(x)\right\}+\left\{X, \underline{l}^{(2)}(x), \bar{l}^{(2)}(x)\right\}= \\
\left\{X, \underline{l}^{(1)}(x)+\underline{l}^{(2)}(x), \bar{l}^{(1)}(x)+\bar{l}^{(2)}(x)\right\}, \\
\left\{X, \underline{l}^{(1)}(x), \bar{l}^{(1)}(x)\right\}-\left\{X, \underline{l}^{(2)}(x), \bar{l}^{(2)}(x)\right\}=
\end{gathered}
$$


Сенъо П

$$
\left\{X, \underline{l}^{(1)}(x)-\bar{l}^{(2)}(x), \bar{l}^{(1)}(x)-\underline{l}^{(2)}(x)\right\},
$$

де $\underline{l}^{(1)}(x), \bar{l}^{(1)}(x), \underline{l}^{(2)}(x), \bar{l}^{(2)}(x),-$ лінійні функції аргументу $x \in R^{n}$.

Однак скористатися безпосередньо відповідними формулами з [3] для означення операцій множення та ділення елементарних лінійних функціональних інтервалів неможливо, бо ми хочемо, щоб результати цих операцій також були лінійними функціональними інтервалами.

Означення 3.4. Нехай $* \in\{+,-, \times, /\}$ - бінарна операція на множині дійсних чисел. Тоді

$$
F(X) * G(X)=\left\{h(x)=f(x) * g(x) \mid f(x) \in F(X), g(x) \in G(X), x \in R^{n}\right\}
$$

визначае відповідну арифметичну операчію над функиіональними інтервалами $F(X) i G(X)$.

Врахувавши означення функціонального інтервалу, поняття його перерізів, реалізацій, властивості нерівностей та означення арифметичних операцій над одновимірними функціональними інтервалами, арифметичні операції над багатовимірними функціональними інтервалами можна виразити за допомогою відповідних операцій над їхніми обмежувальними функціями.

3 погляду реалізації функції $f\left(x_{1}, x_{2}, \ldots, x_{n}\right)$ за допомогою комп'ютера, кожна функція багатьох змінних $\epsilon$ скінченною кількістю арифметичних операцій над деякими елементарними, типовими, спеціальними функціями однієї змінної $\varphi^{(i)}\left(x_{j}\right), \quad(i=1, \ldots, \quad N ; j \in\{1,2, \ldots, n\}$, числами та суперпозицій таких функцій. Властивості всіх таких функцій нам цілком відомі. Зокрема, нам відомі всі характерні точки кожної такої функції $\varphi^{(i)}\left(x_{j}\right)$ на інтервалі $X_{j}$ (кінці інтервалу $X_{j}$, точки її екстремумів, перегинів, її нулі, точки розривів, точки недиференційовності тощо). Тому для кожної такої функції $\varphi^{(i)}(x)$ на інтервалі $X_{j}$ можна побудувати функціональний інтервал $\Phi^{(i)}\left(X_{j}\right)$, який містить графік цієї функції. Виконавши всі арифметичні операції над функціональними інтервалами $\Phi^{(i)}\left(X_{j}\right)$ всіх тих функцій $\varphi^{(i)}\left(x_{j}\right)$, які входять до аналітичного виразу функції $f\left(x_{1}, x_{2}, \ldots, x_{n}\right)$, отримаємо іiї функціональний інтервал на всьому інтервалі $X=X_{1} \times X_{2} \times \ldots X_{n}$.

Однак для практичного застосування доцільно використовувати інші означення багатовимірних функціональних інтервалів, і операцій над ними. Це породжено тим, під час побудови багатовимірного функціонального інтервалу функції багатьох змінних потрібно виконувати операції над багатовимірними функціональними інтервалами різної вимірності. 3 огляду на це побудову багатовимірних функціональних інтервалів і операції над ними будемо реалізовувати такими методами.

1. Перший метод полягає у використанні методу декомпозиції та спрощеної математики функціональних інтервалів у $R^{1}$. Реалізуючи їх, виділяємо з аналітичного виразу цієї функції $f\left(x_{1}, x_{2}, \ldots, x_{n}\right)$ множини $\left\{\varphi_{i 1}\left(x_{i}\right), \varphi_{i 2}\left(x_{i}\right), \ldots, \varphi_{i k_{i}}\left(x_{i}\right)\right\}$ функцій $\varphi_{i j}\left(x_{i}\right)$ лише одного аргументу $x_{i},(i=\overline{1, n})$ та застосовуємо для побудови функціонального інтервалу $\Phi_{i j}\left(X_{i}\right)=\left\{X_{i}, \underline{c}_{i j}\left(x_{i}\right), \bar{c}_{i j}\left(x_{i}\right)\right\}$ кожної функції $\varphi_{i j}\left(x_{i}\right)$, $\left(j=\overline{1, k_{i}}\right)$ кожної такої множини функцій спрощеної математики функціональних інтервалів у $R^{1}$. Тут $X_{i}=\bigcup_{k=1}^{m_{i j}-1} X_{i j k}=\bigcup_{k=1}^{m_{i j}-1}\left[\underline{x}_{i j k}, \bar{x}_{i j k}\right], m_{i j}$ - кількість характерних точок функції $\varphi_{i j}\left(x_{i}\right)$ у інтервалі $X_{i}, \underline{x}_{i j k}, \bar{x}_{i j k}-$ їі суміжні характерні точки, $\underline{c}_{i j}\left(x_{i}\right), \bar{c}_{i j}\left(x_{i}\right)$ - кусково-постійні функції аргументу $x_{i}$ : 


$$
\begin{aligned}
& \bar{c}_{i j}\left(x_{i}\right)=\left\{\begin{array}{c}
\bar{c}_{i j 1}, \text { дкщо } x_{i j 1} \leq x_{i}<x_{i j 2}, \\
\bar{c}_{i j 2}, \text { дкщо } x_{i j 2} \leq x_{i}<x_{i j 3}, \\
\vdots \\
\bar{c}_{i j\left(m_{i j}-1\right)}, \operatorname{s\kappa що~} x_{i j\left(m_{i j}-2\right)} \leq x<x_{i j\left(m_{i j}-1\right)},
\end{array}\right. \\
& \underline{c}_{i j}\left(x_{i}\right)=\left\{\begin{array}{c}
\underline{c}_{i j 1}, \text { лкщо } x_{i j 1} \leq x_{i}<x_{i j 2}, \\
\underline{c}_{i j 2}, \operatorname{s\kappa що~} x_{i j 2} \leq x_{i}<x_{i j 3}, \\
\vdots \\
\underline{c}_{i j\left(m_{i j}-1\right)}, \operatorname{s\kappa що~} x_{i j\left(m_{i j}-2\right)} \leq x_{i}<x_{i j\left(m_{i j}-1\right)},
\end{array}\right.
\end{aligned}
$$

$\underline{c}_{i j k}, \bar{c}_{i j k}-$ відповідні константи. Нехай у результаті розбиття інтервалів $X_{i}$ точками об'єднаної множини характерних точок всіх функцій $\varphi_{i j}\left(x_{i}\right)$ отримані інтервали $X_{i 1}, X_{i 2}, \ldots, X_{i r_{i}}$. Отже, інтервал $X=X_{1} \times X_{2} \times \ldots \times X_{n}$ розбитий характерними точками цих функцій на інтервали $X_{i_{1} i_{2} \ldots i_{n}}=X_{1 i_{1}} \times X_{2 i_{2}} \times \ldots \times X_{n i_{n}}$, де $i_{j}=\overline{1, r_{j}}, \quad(j=\overline{1, n})$, кожен із яких $€$ декартовим добутком інтервалів, межі яких суміжні характерні точки об'єднаної множини характерних точок функцій $\varphi_{i j}\left(x_{i}\right)$, відповідно. Оскільки аргументи функції $f\left(x_{1}, x_{2}, \ldots, x_{n}\right)$ незалежні змінні і значення функції $\varphi_{i j}$ визначаються лише значенням аргументу $x_{i}$, то у всіх точках всіх просторів, які мають вимір $x_{i}$, координата за якою $\tilde{x}_{i}$, зокрема і у всіх точках інтервалу $X_{i_{1} i_{2} \ldots i_{n}}$, значення функції $\varphi_{i j} \in \varphi_{i j}\left(\tilde{x}_{i}\right)$. Згідно з (3), (4) функціональний інтервал кожної функції $\varphi_{i j}\left(x_{i}\right)$ на інтервалі $X_{i j_{i}} \subseteq X_{i j k}$, а отже, і у всьому інтервалі $X_{i_{1} i_{2} \ldots i_{n}}, \epsilon$ тим самим інтервалом $\left[\underline{c}_{i j k}, \bar{c}_{i j k}\right]$, відповідно. Тому функціональний інтервал $F\left(X_{i_{1} i_{2} \ldots i_{n}}\right)=\left\{X_{1 i_{1}} \times X_{2 i_{2}} \times \ldots \times X_{n i_{n}}, \underline{c}_{i_{1} i_{2} \ldots i_{n}}, \bar{c}_{i_{1} i_{2} \ldots i_{n}}\right\}$ функції $f\left(x_{1}\right.$, $\left.x_{2}, \ldots, x_{n}\right)$ на кожному інтервалі $X_{i_{1} i_{2} \ldots i_{n}}=X_{1 i_{1}} \times X_{2 i_{2}} \times \ldots \times X_{n i_{n}}$, де $i_{j}=$ $\overline{1, r_{j}}$, отримуємо результат виконання відповідних операцій, згідно з їі аналітичним виразом, замінивши попередньо у ньому на інтервалі $X_{i_{1} i_{2} \ldots i_{n}}$, такому що $X_{i j_{i}} \subseteq X_{i j k}$, кожну функцію $\varphi_{i j}\left(x_{i}\right)$ інтервалом $\left[\underline{c}_{i j k}, \bar{c}_{i j k}\right]$. Тут на кожному інтервалі $X_{i_{1} i_{2} \ldots i_{n}}$ константи $\underline{c}_{i_{1} i_{2} \ldots i_{n}}, \bar{c}_{i_{1} i_{2} \ldots i_{n}}$ це кінці інтервалу $\left[\underline{c}_{i_{1} i_{2} \ldots i_{n}}, \bar{c}_{i_{1} i_{2} \ldots i_{n}}\right]$, який отримуємо внаслідок цих обчислень.

2. Другий метод полягає у використанні методу декомпозиції, спрощеної математики функціональних інтервалів та повноцінної математики функціональних інтервалів. У цьому випадку, як і у попередньому методі, виділяємо з аналітичного виразу заданої функції $f\left(x_{1}, x_{2}, \ldots, x_{n}\right)$ множини $\left\{\varphi_{i 1}\left(x_{i}\right), \varphi_{i 2}\left(x_{i}\right), \ldots, \varphi_{i k_{i}}\left(x_{i}\right)\right\}$ функцій $\varphi_{i j}\left(x_{i}\right)$ лише одного аргументу $x_{i},(i=\overline{1, n})$ і застосовуємо для побудови функціонального інтервалу $\Phi_{i j}\left(X_{i}\right)=\left\{X_{i}, \underline{c}_{i j}\left(x_{i}\right), \bar{c}_{i j}\left(x_{i}\right)\right\}$ кожної функції $\varphi_{i j}\left(x_{i}\right)$, $\left(j=\overline{1, k_{i}}\right)$ кожної такої множини функцій спрощеної математики функціональних інтервалів у $R^{1}$ та лінійні функціональні інтервали $L \Phi_{i j}\left(X_{i}\right)=\left\{X_{i}, \underline{l}_{i j}\left(x_{i}\right), \bar{l}_{i j}\left(x_{i}\right)\right\}$ цих самих функцій за повноцінною математикою функціональних інтервалів у $R^{1}$. Тут $X_{i}, m_{i}, \underline{x}_{i j k}, \bar{x}_{i j k}, \underline{c}_{i j}\left(x_{i}\right), \bar{c}_{i j}\left(x_{i}\right), \underline{c}_{i j k}, \bar{c}_{i j k}$ ті ж, що і у першому методі, $\underline{l}_{i j}\left(x_{i}\right), \bar{l}_{i j}\left(x_{i}\right)$ - кусково-лінійні функції аргументу $x_{i}$

$$
\bar{l}_{i j}\left(x_{i}\right)=\left\{\begin{array}{c}
\bar{l}_{i j 1}\left(x_{i}\right), \text { дкщо } x_{i j 1} \leq x_{i}<x_{i j 2}, \\
\bar{l}_{i j 2}\left(x_{i}\right), \text { дкщо } x_{i j 2} \leq x_{i}<x_{i j 3}, \\
\vdots \\
\bar{l}_{i j\left(m_{i j}-1\right)}\left(x_{i}\right), \text { дкщо } x_{i j\left(m_{i j}-2\right)} \leq x<x_{i j\left(m_{i j}-1\right)}
\end{array}\right.
$$




$$
\underline{l}_{i j}\left(x_{i}\right)=\left\{\begin{array}{c}
\underline{l}_{i j 1}\left(x_{i}\right), \text { лкщо } x_{i j 1} \leq x_{i}<x_{i j 2}, \\
\underline{l}_{i j 2}\left(x_{i}\right), \text { лкщо } x_{i j 2} \leq x_{i}<x_{i j 3}, \\
\vdots \\
\underline{l}_{i j\left(m_{i j}-1\right)}\left(x_{i}\right), \text { дкщо } x_{i j\left(m_{i j}-2\right)} \leq x_{i}<x_{i j\left(m_{i j}-1\right)},
\end{array}\right.
$$

$\underline{l}_{i j k}\left(x_{i}\right), \bar{l}_{i j k}\left(x_{i}\right)$ - відповідні лінійні функції.

Через розбиття інтервалів $X_{i}$ точками об'єднаної множини характерних точок всіх функцій $\varphi_{i j}\left(x_{i}\right)$ отримуємо інтервали $X_{i 1}, X_{i 2}, \ldots, X_{i r_{i}}$. Тому інтервал $X=$ $X_{1} \times X_{2} \times \ldots \times X_{n}$ розбитий характерними точками цих функцій на інтервали $X_{i_{1} i_{2} \ldots i_{n}}=X_{1 i_{1}} \times X_{2 i_{2}} \times \ldots \times X_{n i_{n}}$, де $i_{j}=\overline{1, r_{j}},(j=\overline{1, n})$, кожен із яких $\epsilon$ декартовим добутком інтервалів, межі яких суміжні характерні точки об'єднаної множини характерних точок функцій $\varphi_{i j}\left(x_{i}\right)$, відповідно.

Згідно з (3), (4), спрощений функціональний інтервал кожної функції $\varphi_{i j}\left(x_{i}\right)$ на інтервалі $X_{i j_{i}} \subseteq X_{i j k}$, а отже, і у всьому інтервалі $X_{i_{1} i_{2} \ldots i_{n}}, \epsilon$ тим самим інтервалом $\left[\underline{c}_{i j k}, \bar{c}_{i j k}\right]$, відповідно. Згідно з $(5),(6)$ повноцінний функціональний інтервал кожної функції $\varphi_{i j}\left(x_{i}\right)$ на тому ж інтервалі $X_{i j_{i}} \subseteq X_{i j k}$, а отже, і у всьому інтервалі $X_{i_{1} i_{2} \ldots i_{n}}$, є тим самим елементарним лінійним функціональним інтервалом $\left[\underline{l}_{i j k}, \bar{l}_{i j k}\right]$, відповідно. Побудуємо на кожному інтервалі $X_{i_{1} i_{2} \ldots i_{n}}=X_{1 i_{1}} \times X_{2 i_{2}} \times$ $\ldots \times X_{n i_{n}}$, де $i_{j}=\overline{1, r_{j}}$, множину гібридних лінійних функціональних інтервалів $\left\{L F_{1}\left(X_{i_{1} i_{2} \cdots i_{n}}\right), L F_{2}\left(X_{i_{1} i_{2} \ldots i_{n}}\right), \ldots, L F_{n}\left(X_{i_{1} i_{2} \ldots i_{n}}\right)\right\}$ функції $z=f\left(x_{1}, x_{2}, \ldots, x_{n}\right)$, де $L F_{s}\left(X_{i_{1} i_{2} \ldots i_{n}}\right)=\left\{X_{1 i_{1}} \times X_{2 i_{2}} \times \ldots \times X_{n i_{n}}, \underline{l}_{i_{1} i_{2} \ldots i_{n}}, \bar{l}_{i_{1} i_{2} \ldots i_{n}}\right\},(s=\overline{1, n})$. Кожен функціональний інтервал $L F_{s}\left(X_{i_{1} i_{2} \ldots i_{n}}\right)$ знаходимо внаслідок виконання відповідних операцій, згідно з аналітичним виразом функції $z=f\left(x_{1}, x_{2}, \ldots, x_{n}\right)$, замінивши попередньо у ньому на інтервалі $X_{i_{1} i_{2} \ldots i_{n}}$, такому що $X_{i j_{i}} \subseteq X_{i j k}$, кожну функцію $\varphi_{j}^{(i)}\left(x_{i}\right)$, коли $i \neq s$, відповідним інтервалом $\left[\underline{c}_{i j k}, \bar{c}_{i j k}\right]$, а кожну функцію $\varphi_{j}^{(s)}\left(x_{i}\right)$ - елементарним лінійним функціональним інтервалом $\left[\underline{l}_{i j k}, \bar{l}_{i j k}\right]$.

Зауваження 3.2. Оскільки $s$ зафіксоване, то лінійний функціональний інтервал $L F_{s}\left(X_{i_{1} i_{2} \ldots i_{n}}\right)$ отримуємо, виконуючи відповідні операції математики функціональних інтервалів у $R^{1}$. Тому цей функціональний інтервал $є$ поширенням так отриманого результату на весь інтервал $X_{i_{1} i_{2} \ldots i_{n}}$.

Зокрема, при $n=2$ графіки обмежувальних функцій $\underline{l}_{i_{1} i_{2}}, \bar{l}_{i_{1} i_{2}}$ функціональних інтервалів $L F_{s}\left(X_{i_{1} i_{2}}\right)=\left\{X_{1 i_{1}} \times X_{2 i_{2}}, \underline{l}_{i_{1} i_{2}}, \bar{l}_{i_{1} i_{2}}\right\}$ на кожному інтервалі $X_{i_{1} i_{2}}=$ $X_{1 i_{1}} \times X_{2 i_{2}}$ при кожному $s=1,2$ є сукупністю відповідних площин, перпендикулярних координатній площині $Z O X_{s}$, де $z=f\left(x_{1}, x_{2}\right)$.

Зауваження 3.3. Оскільки кожен функціональний інтервал $L F_{s}\left(X_{i_{1} i_{2} \ldots i_{n}}\right) \epsilon$ лінійним функціональним інтервалом тієї самої функції $z=f\left(x_{1}, x_{2}, \ldots, x_{n}\right)$, то i перетин всіх цих функціональних інтервалів та будь-яких вибірок із них є лінійними функціональними інтервалами цієї ж функції.

Для знаходження таких перетинів потрібно розв'язувати системи відповідних лінійних алгебричних рівнянь. Очевидно, що функціональний інтервал, який $є$ перетином всіх функціональних інтервалів $L F_{s}\left(X_{i_{1} i_{2} \ldots i_{n}}\right),(s=\overline{1, n})$, має найменшу ширину.

Оскільки $\bigcup_{i_{n}=1}^{r_{n}} \ldots \bigcup_{i_{2}=1}^{r_{2}} \bigcup_{i_{1}=1}^{r_{1}} X_{i_{1} i_{2} \ldots i_{n}}=X$, то при всіх $s=\overline{1, n}$ кожне об'єднання $\overline{L F}_{s}(X)=\bigcup_{i_{n}=1}^{r_{n}} \ldots \bigcup_{i_{2}=1}^{r_{2}} \bigcup_{i_{1}=1}^{r_{1}} L F_{s}\left(X_{i_{1} i_{2} \ldots i_{n}}\right)$ є лінійним функціональним інтервалом функції $z=f\left(x_{1}, x_{2}, \ldots, x_{n}\right)$ на інтервалі $X=X_{1} \times X_{2} \times \ldots \times X_{n}$. $\mathrm{Y}$ різних $s$ це різні лінійні функціональні інтервали. Лінійними функціональними 
інтервалами функції $z=f\left(x_{1}, x_{2}, \ldots, x_{n}\right)$ на цьому ж інтервалі $\epsilon$ і перетини всіх цих функціональних інтервалів та будь-яких вибірок із них. Для знаходження таких перетинів також потрібно розв'язувати системи відповідних лінійних алгебричних рівнянь. Очевидно, що функціональний інтервал, який є перетином всіх функціональних інтервалів $\overline{L F}_{s}(X),(s=\overline{1, n})$, має найменшу ширину.

Так побудовані лінійні функціональні інтервали функції $f\left(x_{1}, x_{2}, \ldots, x_{n}\right)$ дають змогу розв'язувати задачі на інтервалі $X=X_{1} \times X_{2} \times \ldots \times X_{n}$, пов'язані 3 нею, зводячи їх до послідовності цих самих, але одновимірних задач по кожній координаті, розв'язуючи їх засобами математики функціональних інтервалів у $R^{1}$.

В [3] запропонований загальний алгоритм розв'язування задачі у $R^{n}$ за допомогою математики функціональних інтервалів з врахуванням її специфіки. Він такий.

Алгоритм 3.1. Нехай $x_{i k}$, де $i=\overline{1, n}, k=\overline{1, r_{i}+1}$, - всі характерні точки об'єднаної множини характерних точок всіх елементарних функцій $\varphi_{j}^{(i)}\left(x_{i}\right)$ аргументу $x_{i}$ із аналітичного виразу функції $f\left(x_{1}, x_{2}, \ldots, x_{n}\right), r_{i}+1$ - їня кількість; $X_{i 1}, X_{i 2}, \ldots, X_{i r_{i}}$ - інтервали, на які розбитий інтервал $X_{i}$ цими характерними точками. Тоді інтервал $X=X_{1} \times X_{2} \times \ldots \times X_{n}$ розбитий на $N=r_{1} r_{2} \ldots r_{n}$ інтервалів $X_{i_{1} i_{2} \ldots i_{n}}$, де $X_{i_{1} i_{2} \ldots i_{n}}=X_{1 i_{1}} \times X_{2 i_{2}} \times \ldots \times X_{n i_{n}}$.

1. По кожній координаті будуємо функціональні інтервали $\Phi_{i j}\left(X_{i}\right)=\left\{X_{i}, \underline{c}_{i j}\left(x_{i}\right), \bar{c}_{i j}\left(x_{i}\right)\right\}$ кожної функцї̈ $\varphi_{i j}\left(x_{i}\right),\left(j=\overline{1, k_{i}}\right)$, застосовуючи спрощену математику функціональних інтервалів у $R^{1}$.

2. Послідовно, починаючи з $i_{1}=i_{3}=i_{4}=\ldots=i_{n}=1$, вибираємо $X_{1 i_{1}}, X_{3 i_{3}}, X_{4 i_{4}}, \ldots, X_{n i_{n}}$ та інтервали

$$
\left[\underline{c}_{1 j i_{1}}, \bar{c}_{1 j i_{1}}\right],\left[\underline{c}_{3 j i_{3}}, \bar{c}_{3 j i_{3}}\right],\left[\underline{c}_{4 j i_{4}}, \bar{c}_{4 j i_{4}}\right], \ldots,\left[\underline{c}_{n j i_{n}}, \bar{c}_{n j i_{n}}\right] .
$$

3. Будуємо функціональні інтервали $\Phi_{2 j}\left(X_{2}\right)=\left\{X_{2}, \underline{c}_{2 j}\left(x_{2}\right), \bar{c}_{2 j}\left(x_{2}\right)\right\}$ кожноӥ функиії $\varphi_{2 j}\left(x_{2}\right),\left(j=\overline{1, k_{2}}\right)$, застосовуючи повноцінну математику функиіональних інтервалів у $R^{1}$.

4. Замінивши в аналітичному виразі функції $f\left(x_{1}, x_{2}, \ldots, x_{n}\right)$ кожну функцію $\varphi_{i j}\left(x_{i}\right), i=1,3,4, \ldots, n,\left(j=\overline{1, k_{i}}\right)$ відповідним інтервалом (7), а функції $\varphi_{2 j}\left(x_{2}\right),\left(j=\overline{1, k_{2}}\right)$ - їхніми лінійними функціональними інтервалами, згідно $з$ (5), (6), та виконавши відповідні операціі, отримаємо лінійний функціональний інтервал $L F\left(X_{2}\right)$ у $R^{1}$.

5. Сформульовану задачу (як одновимірну) розв'язуємо за допомогою цього лінійного функціонального інтервалу. Розв'язком їі буде один, чи декілька інтервалів із множини інтервалів $X_{21}, X_{22}, \ldots, X_{2 r_{2}}$ інтервалу $X_{2}$, або їхні частини. Нехай $X_{2}^{1}$ - їнє об'єднання. Тоді розв'язок сформульованої задачі у множині інтервалів $X_{1 i_{2} 11 \ldots 1}$ може бути лише у $X_{11} \times X_{2}^{1} \times X_{31} \times X_{41} \times \ldots \times$ $X_{n 1}$.Тому далі інші інтервали із $X_{1 i_{2} 11 \ldots 1}$ вже не розглядаємо.

6. Будуємо функціональні інтервали $\Phi_{1 j}\left(X_{1}\right)=\left\{X_{1}, \underline{c}_{1 j}\left(x_{1}\right), \bar{c}_{1 j}\left(x_{1}\right)\right\}$ кожної функції $\varphi_{1 j}\left(x_{1}\right),\left(j=\overline{1, k_{1}}\right)$, застосовуючи повноцінну математику функціональних інтервалів у $R^{1}$.

7. Послідовно, починаючи із $i_{3}=i_{4}=\ldots=i_{n}=1$, вибираємо

$$
X_{2}^{1}, X_{3 i_{3}}, X_{4 i_{4}}, \ldots, X_{n i_{n}},
$$

та інтервали

$$
\left[\underline{c}_{2 j i_{1}}^{1}, \bar{c}_{2 j i_{1}}^{1}\right],\left[\underline{c}_{3 j i_{3}}, \bar{c}_{3 j i_{3}}\right],\left[\underline{c}_{4 j i_{4}}, \bar{c}_{4 j i_{4}}\right], \ldots,\left[\underline{c}_{n j i_{n}}, \bar{c}_{n j i_{n}}\right] .
$$


Сенво П

8. Замінивши в аналітичному виразі функції $f\left(x_{1}, x_{2}, \ldots, x_{n}\right)$ кожну функцію $\varphi_{i j}\left(x_{i}\right), i=2,3,4, \ldots, n,\left(j=\overline{1, k_{i}}\right)$ відповідним інтервалом (8), а функції $\varphi_{1 j}\left(x_{1}\right),\left(j=\overline{1, k_{1}}\right)$ - іхніми лінійними функціональними інтервалами, згідно 3 (5), (6), та виконавши відповідні операції, отримаємо лінійний функціональний інтервал $L F\left(X_{1}\right)$ у $R^{1}$.

9. Сформульовану задачу (як одновимірну) розв'язуємо за допомогою цього лінійного функціонального інтервалу. Розв'язком тї буде один, чи декілька інтервалів із множини інтервалів $X_{11}, X_{12}, \ldots, X_{1 r_{1}}$ інтервалу $X_{1}$, або їхні частини. Нехай $X_{1}^{2}$ - іхнє об'єднання, а $\hat{X}_{1}^{2}=X_{1}^{2} \bigcap X_{11}$. Якщо $\hat{X}_{1}^{2}=\varnothing$, то у множині інтервалів $X_{i_{1} 11 \ldots 1}, X_{1 i_{2} 11 \ldots 1}$ розв'язку сформульованої задачі немає. У протилежному випадку розв'язок сформульованої задачі у множині інтервалів $X_{i_{1} 11 \ldots 1}, X_{1 i_{2} 11 \ldots 1}$ може бути лише у $X_{11} \times X_{21} \times X_{31} \times X_{41} \times \ldots \times X_{n 1}$. Тому далі інші інтервали з множини інтервалів $X_{i_{1} 11 \ldots 1}, X_{1 i_{2} 11 \ldots 1}$ вже не розглядаємо.

10. Далі пошук розв'язку цієї задачі продовжуємо за цим алгоритмом для всіх інших пар координат, починаючи з пункту 2 цього алгоритму.

\title{
4. ВИСНОВКИ
}

Розроблене числення на множині багатовимірних функціональних з використанням спрощеної математики функціональних інтервалів у $R^{1}$ дає змогу ефективно розв'язувати задачі у $R^{n}$ при $n>1$ методами математики функціональних інтервалів у $R^{1}$. У цьому випадку треба послідовно застосовувати декомпозицію сформульованої задачі по кожній координаті згідно з алгоритмом 3.1 та в кінці обчислень виконати перетин так отриманих проміжних результатів.

\section{СПИСОК ВИКОРИСТАНОЇ ЛІТЕРАТУРИ}

1. АлефельдГ. Введение в интервальные вычисления /Г. Алефельд, Ю. Херцбергер.Москва: Мир, $1987-357$ с.

2. Сенъо П. С. Арифметика лінійних функціональних інтервалів / П. С. Сеньо // Вісн. Львів. ун-ту. Сер. прикл. матем. та інформ. - 2014.- Вип. 21.- С. 38-57.

3. Senio P.S. Algorithms for Solving Problems using Mathematics of Functional Intervals / P. S. Senio // XXX International Conference Problems of Decision Making Under Uncertainties (PDMU-2019). - Prague. Czech Republic. - Kyiv. - August 27-31.- 2019. - P. 107108.

Стаття: надійшла до редколегії 01.10.2020

доопрацвована 15.10 .2020

прийнята до друку 19.10.2020

\section{SPECIFICS OF MATHEMATICS OF MULTIDIMENSIONAL FUNCTIONAL INTERVALS}

\author{
P. Senio \\ Ivan Franko National University of Lviv, \\ Universytetska str., 1, Lviv, 79000, Ukraine, e-mail:petrosny@ukr.net \\ In this paper it is considered the mathematics of functional intervals $R^{n}, n>1$ devel- \\ oped by the author; it is pointed out the specific moments in it while constructing func- \\ tional intervals of functions of many variables and arithmetic, unary and theoretical and
}


set operations as well as there are presented effective algorithms of their implementation. In this regard there are applied the decomposition method and simplified mathematics of functional intervals in $R^{1}$. This is due to the following. The results of performing arithmetic operations on functional intervals are determined by the corresponding operations on their constraint functions [2]. But the results of performing arithmetic operations on linear functions are the corresponding linear functions only when adding and subtracting such functions. In addition, even the results $R^{2}$, of multiplication and division of such functions may contain, for example, saddle points, which do not allow to approximate them by linear functions. Therefore, in such cases, the set of results of actions on multivariate functional intervals is not closed. In this paper, the problem described is solved by applying simplified mathematics of functional intervals in all coordinates, except one, which is gradually changed from 1 to $n$. Then all arithmetic operations on multidimensional functional intervals are reduced to the corresponding operations on two one-dimensional functional intervals, the limiting functions of one of which are piecewise constant functions. The linear functional interval of this function of many variables on the specified multidimensional interval is the intersection of all so constructed one-dimensional functional intervals and any samples from them. To find such intersections, we need to solve the corresponding systems of linear algebraic equations.

Key words: interval, functional interval, interval extension of function, two-sided approximation, mathematics of functional intervals. 